\title{
New and Emerging Opioid Overdose Risk Factors
}

\author{
Ralph Foglia ${ }^{1} \cdot$ Anna Kline ${ }^{1} \cdot$ Nina A. Cooperman ${ }^{1}$ (D) \\ Accepted: 15 April 2021 / Published online: 22 April 2021 \\ (C) The Author(s), under exclusive licence to Springer Nature Switzerland AG 2021
}

\begin{abstract}
Purpose of Review The purpose of this review is to provide a review of the current literature surrounding opioid overdose risk factors, focusing on relatively new factors in the opioid crisis.

Recent Findings Both a market supply driving force and a subpopulation of people who use opioids actively seeking out fentanyl are contributing to its recent proliferation in the opioid market. Harm reduction techniques such as fentanyl testing strips, naloxone education and distribution, drug sampling behaviors, and supervised injection facilities are all seeing expanded use with increasing amounts of research being published regarding their effectiveness. Availability and use of interventions such as medication for opioid use disorder and peer recovery coaching programs are also on the rise to prevent opioid overdose.

Summary The opioid epidemic is an evolving crisis, necessitating continuing research to identify novel overdose risk factors and the development of new interventions targeting at-risk populations.
\end{abstract}

Keywords Opioid overdose $\cdot$ Risk factors $\cdot$ Naloxone $\cdot$ Fentanyl $\cdot$ Harm reduction

\section{Introduction}

Between 2010 and 2015 [1], deaths in the United States (US) from opioid overdose tripled, and in 2018, the US opioid crisis (US) claimed 128 lives per day [2]. Targeted, efficacious interventions are clearly needed to reduce the rising toll of opioid-related deaths. For preventive public health interventions to succeed, however, they must be based on a clear understanding of the characteristics and risk behaviors of people who use opioids (PWUO) that increase their vulnerability to overdose. Earlier studies and reviews on overdose risk have found positive associations between overdose risk and opioid dosage; comorbid mental health or substance abuse disorders; concurrent use of other sedative drugs, medications, and alcohol; resumed opioid use after a period of abstinence; injection drug use; and inadequately treated chronic pain $[3,4]$. While the identification of these factors is helpful for identifying individuals at risk for opioid overdose, more research is

This article is part of the Topical Collection on Opioids

Nina A. Cooperman

Nina.Cooperman@rutgers.edu

1 Department of Psychiatry, Rutgers Robert Wood Johnson Medical School, 317 George Street, Suite 105, New Brunswick, NJ 08901, USA needed to determine the impact of recently emerging factors such as fentanyl in the drug supply and increasing availability of naloxone (an opioid antagonist that reverses the effects of opioid overdose) [3]. To date, no known reviews describing the literature on opioid overdose risk factors have included factors that are new to the current opioid crisis. Therefore, the goal of this review is to (1) provide a review of the current literature surrounding opioid overdose risk factors, focusing on the influence of relatively new factors in the opioid crisis; (2) address current gaps in knowledge regarding what is known about overdose risk, and (3) suggest future research paths to address these gaps.

\section{Prevalence of Overdose}

The CDC reported that about $70 \%$ of drug overdose deaths in 2018 involved opioids [5]. Opioid overdoses are increasing, on average, $5.6 \%$ per quarter, with models predicting the crisis to worsen to 82,000 deaths by 2025 and 700,000 deaths between 2016 and 2025 [6, 7]. These predicted trends do not take into account the effects of the current covid-19 pandemic, which has coincided with a marked increase in opioid overdose. The Federal Office of National Drug Control Policy reported a spike in overdose reports since the first reported case of covid-19 in the United States, with an average increase 
of 20\% when comparing January-April 2019 and JanuaryApril 2020 [8]. Certain parts of the country have seen even more dramatic spikes in overdose correlating with quarantine due to covid-19. For example, Milwaukee, WI and Franklin County, $\mathrm{OH}$ experienced a $54 \%$ and $50 \%$ increase in fatal overdoses, respectively $[9,10]$.

However, the emergence of fentanyl has perhaps been the most important driver of the current overdose crisis. From 2017 to 2018, the CDC reported a decrease in fatal overdoses due to prescription opioids $(13.5 \%)$ and heroin $(4.1 \%)$, but a $10 \%$ increase in fatal overdoses involving synthetic opioids, including fentanyl and its derivatives [5]. This shift could be due to targeted efforts at changing opioid prescribing practices, leading some individuals to seek illicit drugs, and the proliferation of fentanyl and its derivatives in the heroin supply [11-14].

\section{Fentanyl}

Fentanyl is 50 to 100 times more potent than morphine and can cause respiratory depression and death with very small doses [15]. The increasing presence of fentanyl in the illicit drug supply marks a shift in the drug market [14]. Given its potency, fentanyl use, whether intentional or not, significantly increases the chance for overdose [16-18]. Current data shows that fentanyl use is steadily rising [13, 19]. For example, in New Jersey, reports showed a staggering $949 \%$ increase in fentanyl submissions to New Jersey State Police Office of Forensic Science labs from 2015 to 2019 [20].

A supply and demand model has been used to explain the current surge in fentanyl in the illicit opioid market [21, 22]. The supply argument points to market factors such as low manufacturing costs and ease of distribution, causing fentanyl to become widely available and therefore more widely used, while the demand argument suggests that many people who use opioids (PWUO) actively seek fentanyl, preferring the fentanyl high and/or its cheaper cost relative to unadulterated heroin. According to this latter argument, consumer demand has driven the increasing supply of fentanyl in the illicit drug market. Hempstead and Yildrin, 2016 present a supply-led argument [23]. They suggest that both the decrease in heroin purity and market competition from prescription opioids put pressure on heroin suppliers by threatening their profit margins due to other options available for buyers. This resulted in increased production and distribution of fentanyl as a response, especially in places where prescription opioids are widely available [23]. While supply-side interventions for pharmaceutical opioids, such as "abuse-deterrent" opioid formulations and provider education to reduce opioid prescribing, were successful at reducing overdoses from prescription pills, there is some evidence that this led to an increase in use of other opioids, like heroin and fentanyl [13, 14]. While prescription opioid overdose has been on the decline, the amount of fentanyl available to purchase on the street has rapidly increased. The limiting of other opioids on the market, combined with the rapid increase in available fentanyl, likely led to a situation where heroin laced with fentanyl or fentanyl alone was a cheap and readily available alternative for both dealers and PWUO and facilitated its use in the community. This increase in fentanyl supply, along with a shift to a greater number of people obtaining opioids from the illegal drug market, helped contribute to the greatly increased risk of overdose and skyrocketing number of overdoses in recent years [13, 24].

Although the increase of fentanyl in the drug supply is contributing to increased opioid overdose, demand for fentanyl among people who use drugs is also likely supporting the market [25]. A cross sectional study of 308 PWUD in Baltimore, Boston and Providence found that $27 \%$ of participants preferred fentanyl to nonadulterated heroin [26••]. Other smaller studies have also demonstrated that there is a population that desires fentanyl- in one study that took place in Dayton, $\mathrm{OH}$ between 2017 and $2018(n=60), 40 \%$ indicated a preference for fentanyl over heroin [27]. While the current policy emphasis for addressing the opioid epidemic has focused on reducing prescription opioid availability, focus has also shifted to interventions aimed at decreasing the demand for opioids and fentanyl, such as engaging and retaining patients with OUD in evidence-based treatment, especially medication treatment [28]. Additionally, public health authorities are also attempting to implement harm reduction strategies to mitigate the risk of overdose among those resistant to addiction treatment.

Fentanyl is a relatively new development in the opioid crisis; yet, it is also critical to understand the role fentanyl plays and interacts with other previously identified risk factors. For example, while factors such as polydrug use have been shown to increase risk of overdose [29, 30] they may also increase an individual's risk of exposure to fentanyl [31]. While also an individual risk factor for overdose, mental illness is associated with increased risky drug use, potentially increasing an individual's exposure to fentanyl [32]. There is extremely limited data on the relationship between opioid overdose and suicide, due to both the likely underreporting of suicide attempts in the context of an overdose and difficulty determining an overdose victim's intent postmortem [33]. Cross-sectional data has shown that over a third $(36 \%)$ of overdose survivors had a strong desire to die (>7/10) before their last overdose. This is concerning given that $92 \%$ used heroin and/or fentanyl and the increased potency of the latter makes overdose significantly more likely [34•]. While these previously identified risk factors are still influencing the opioid crisis, more research is needed to identify exactly how they fit in with the newer factors affecting the current opioid climate. Determining the drivers of the fentanyl crisis and 
implementing different interventions that variably impact these factors will play a pivotal role in addressing the ongoing crisis.

\section{Harm Reduction}

\section{Naloxone}

Naloxone is a [mu opioid receptor antagonist used to reverse opioid overdose by competing with opioids for receptor binding. While first responders and clinicians have been utilizing naloxone to reverse opioid overdose for decades, efforts to expand naloxone availability through prescription take-home naloxone and pharmacy dispensing have culminated in all 50 states passing laws allowing pharmacy-based naloxone dispensing [35]. Most states have also expanded these laws to include two ways of obtaining naloxone without a traditional "provider-patient" relationship - laws where providers can write naloxone prescriptions for friends or family members of someone at risk of overdose and laws permitting pharmacists to dispense naloxone on a standing, nonpatient specific order [36]. Additionally, community-based programs aimed at naloxone education and distribution have become increasingly important, allowing lay persons to obtain naloxone even without a prescription [37].

Studies evaluating naloxone have demonstrated its effectiveness for saving lives and the willingness of people to use it [38 $]$. A phone interview study of patients prescribed opioids for pain in Australia ( $n=208)$ showed that the majority $(60 \%)$ thought that medical providers offering naloxone to people prescribed opioids was a good or very good idea [39]. Qualitative studies utilizing semistructured interviews such as the one performed by Mueller et al. ( $n=24)$ attempt to uncover factors that facilitate or serve as barriers to a patient accepting a naloxone prescription when prescribed opioids in a primary care setting [40]. While factors such as nonjudgmental communication from providers and providing education about both opioids and naloxone increased acceptance of naloxone, barriers to naloxone acceptance included limited knowledge of naloxone among patients and patients' fear of enhancing their providers concerns about opioid abuse or misuse [40].

In a study analyzing national data from 2005 to 2016, naloxone access laws giving pharmacists permission to distribute naloxone (either through granting the pharmacist prescriptive authority or allowing pharmacies to distribute naloxone without a prescription) have been estimated to have decreased opioid overdose deaths by $27 \%$ and $34 \%$ in their second and third year after adoption, respectively [41]. However, this study was limited to dispensing data from the Medicaid population which may not represent overall changes in naloxone access. Another study drawing on 2000-2014 data from the
National Vital Statistics System and the National Survey on Drug use and Health found naloxone access and Good Samaritan laws (laws that protect individuals reporting overdose from arrest and prosecution surrounding illicit substance use, possession, or violation of probation) both demonstrated reductions in opioid overdose deaths $[42 \bullet \cdot]$. These effects were shown to be most pronounced in the African American population with naloxone access decreasing deaths by $23 \%$ (vs. $14 \%$ in the general population) and good Samaritan laws leading to a $26 \%$ reduction (vs. $15 \%$ in the general population) [43]. Importantly, this study also found no increase in nonmedical opioid use after both naloxone access and Good Samaritan laws were passed. While this study's use of largescale surveys and administrative data allowed investigators to analyze a large number of participants and follow state level trends, the study was limited by its observational design which precluded conclusions about causality. Modeling studies using United Kingdom data have also shown naloxone to be a cost-effective way to combat the opioid crisis [43]. Yet, diagnosing and coding of opioid overdose is often inconsistent in administrative records, leading to the likelihood that that opioid overdose is underestimated [44].

Prospective studies have also examined the efficacy of take-home naloxone to prevent overdose. One prospective cohort study enrolled 244 participants from opioid treatment programs with a diagnosis of OUD and provided them with naloxone education, training, and two doses of take-home naloxone. After 3 months of follow up, 31 of the participants reported using the naloxone to reverse overdose [45]. This validated both the concept that PWUO are willing to administer naloxone and, importantly, that they are often acting in a "bystander" capacity where the recipient of the naloxone is using the drug to rescue someone else (only one participant in the study overdosed during the study period, and they themselves had previously rescued someone). This speaks to the efficacy of naloxone as a harm reduction tool where PWUO provided with naloxone can facilitate the rescue of others in the community. Another larger $(n=351)$ prospective observational study in New York City found that over a year, in 241 overdoses witnessed by individuals who completed overdose training and were provided naloxone, naloxone was administered in $77 \%$ of witnessed overdoses $(60 \%$ of the time provided by the study participant themselves) [46]. A prospective trial of 395 individuals in New Mexico who were provided overdose education and take-home naloxone demonstrated 114 overdose reversals in the following year [47]. It is important to note that in all of these studies, overdose rescues can only be fully measured on participants with complete follow up and are therefore likely underestimated. A county-level study in North Carolina showed not only decreased opioid deaths after the implementation of naloxone kit distribution programs but demonstrated their cost effectiveness (saving approximately $\$ 2700$ for every dollar used obtaining and 
distributing the kits) [48]. On a population level, a time series analysis by Walley et. al. found that communities that had even 1-100 enrollments per 100,000 population in an opioid education and naloxone distribution program had significantly decreased overdose deaths compared to communities without the programs (adjusted rate ratio 0.73 , 95\% CI 0.57-0.91) [49]. Together, there is a large body of observational data that suggests take-home naloxone is a successful harm reduction technique for reducing overdose deaths. However, because all such studies to date have been observational, their results cannot be taken to infer a causal connection between increased naloxone accessibility and reduced overdose mortality. While randomized control trials would be the gold standard, ethical considerations preclude their use since randomization would require withholding a potentially lifesaving drug from a population that needs it. Moreover, it is impossible to quantify the mortality benefits of many of these observational studies since one cannot determine how many individuals who received naloxone from a study participant would have received naloxone had the study participant not been there (i.e., from another bystander, an EMT/police officer, etc.). It is also impossible to know how many of the rescued participants would have survived the overdose had naloxone not been administered.

Some individuals have expressed concerns that take home naloxone provides a "safety net" for PWUO, encouraging riskier opioid use and discouraging PWUO from quitting $[50,51]$. A qualitative study by Heavey et al. $(n=20)$, for example, found participants reporting high risk behavior in the presence of naloxone, such as using higher quantities of drugs and, in one instance, intentional fentanyl use [52]. Another qualitative study examining current past and present opioid users in New Jersey found that about half of the sample $(N=36)$ believed increased naloxone access led to riskier drug behavior, including intentional fentanyl use [53]. However, many of the participants expressed this about others without endorsing increased risk behavior themselves. Other studies have suggested that naloxone does not lead to increased opioid use [54]. One study of ED patients at an urban level one trauma center $(n=89)$, for example, showed that there was increased opioid use in only one participant (4\%) while there was a decrease in nine (33\%), and take-home naloxone increased treatment engagement [55]. Another study based in Los Angeles, California found that after a 1-h training session in recognizing overdose and administering naloxone, 25 (53\%) of the 47 participants who responded to a 3-month follow-up survey had decreased their personal drug use after receiving the training [56]. Given the mixed results of small studies examining the impact of naloxone on opioid risk behavior, further research and larger studies are needed to determine the true impact of naloxone availability on opioid risk behavior.

Determining an appropriate naloxone dose is challenging. Given that the half-life of naloxone is typically shorter than many opioids (less than $30 \mathrm{~min}$ ), individuals can potentially reoverdose on opioids without an additional dose of naloxone $[57,58]$. This problem is compounded by the increasing use of fentanyl and its derivatives, which require an increased amount of naloxone to achieve a reversal. Further clinical challenges arise when those providing naloxone are not aware whether fentanyl was used or not [59]. Also, since naloxone is a pure mu-opioid receptor antagonist, it can precipitate acute opioid withdrawal symptoms [57]. In qualitative studies, researchers found that, individuals frequently complained of naloxone-induced withdrawal symptoms, leading to a number of adverse medical outcomes [60]. Thus, at least three qualitative studies describe overdose survivors attempting to reuse illicit opioids immediately after emergency room discharge to relieve the symptoms of withdrawal. Further, some individuals couldn't wait to leave the hospital to relieve their withdrawal symptoms and used opioids while still in the hospital $[53,60,61]$. Further, the desire to avoid withdrawal symptoms may discourage overdose survivors from seeking naloxone resuscitation or medical care $[53,61]$. Additional studies determining the prevalence and distribution of these attitudes and behaviors in the community and research focusing on optimal naloxone dosing will likely optimize the benefits of naloxone. Programs focused on opioid and naloxone education that can provide PWUO information on the benefits of naloxone, as well as correct information on its pharmacological effects, can increase uptake. Further, increased assessment and treatment of opioid withdrawal symptoms postopioid overdose and naloxone reversal by medical professionals could decrease treatment-avoiding behavior and opioid reuse after naloxone administration.

Despite general support for naloxone distribution among public health professionals and clinicians [62], naloxone is still not widely accessible to all. The price of naloxone continues to be a barrier to its access, suggesting that those without means of purchasing it or obtaining it through subsidized naloxone distribution programs could be at an increased risk of fatal overdose [63]. Studies analyzing geographic regions show that some of the highest risk areas do not have adequate access to naloxone in their pharmacies, minimizing its availability even if community members have the means of purchasing the life-saving drug [64]. Another study based in Erie County, NY analyzing 198 participants in opioid education and naloxone distribution (OEND) programs found that none of the participants had personally experienced an overdose, and only $14 \%$ had witnessed an overdose, suggesting that the programs may have been attracting concerned members of the community but not necessarily penetrating into the highest risk population [65]. However, other research surveying organizations that distributed over 150,000 naloxone kits from 1996 to 2014 has shown that $81.6 \%$ of people receiving naloxone kits were people who used drugs and $81.6 \%$ of overdoses reversed involved heroin, suggesting that naloxone 
kits were being distributed to the population at risk [37]. Eliminating barriers and continuing to expand naloxone access to anyone at risk remains a high priority public health goal in minimizing overdose.

\section{Supervised Drug Consumption}

Using opioids alone is a risk factor for fatal overdose [66]. Given that an individual experiencing an opioid overdose is usually nonresponsive or unconscious, another individual needs to be present to administer naloxone to reverse the overdose. Studies from British Columbia show that the majority of overdose deaths involve users who use drugs alone and indoors, minimizing their chances of being found and receiving assistance in case of an overdose [67]. In fact, most fatal overdoses occur without any connection to health services, help, or ambulance call, further suggesting that fatal overdoses usually occur among people using alone [68]. While it is known that using alone increases risk of fatality in the context of an overdose, using alone remains prevalent often due to the stigma and shame associated with drug use [69].

The risk of overdose associated with using alone may be mitigated by the establishment of supervised injection sites, in which PWUO consume drugs in the presence of trained observers who offer medical assistance in the case of overdose or other adverse events. Although not currently operating in the US, supervised injection or consumption facilities exist in other countries and are known to be an effective harm reduction strategy for reducing fatal overdose [70]. These facilities are "safe spaces" for drug users where they can use drugs freely without fear of incarceration or stigmatization, often in the presence of trained medical professionals who can administer naloxone, if necessary [71]. Supervised consumption facilities currently operate in 11 countries, with no known fatal overdoses occurring at these sites [70]. While legal supervised injection facilities are not yet available in the US, several facilities have been proposed across the country [72]. Vancouver, CN not only demonstrated a $35 \%$ reduction in overdose mortality after the implementation of safe consumption spaces but increased enrollment in drug treatment directly resulting from use of these spaces [73]. Studies assessing willingness to use drugs in these safe spaces, among people who use drugs, have found that $77 \%$ of 175 participants in Baltimore, and $91 \%$ of 237 participants in Boston expressed a willingness to use the spaces if they were made available $[74,75]$. These same studies also assessed perceived barriers to utilization of these safe spaces, and found that the barriers included concerns about arrest, privacy, and transportation cost associated with getting to and using these locations. Overall, safe consumption spaces appear to be effective harm reduction tools to minimize fatal overdose in other countries and have the potential to save lives, if they legally become available, in the US.

\section{Fentanyl Test Strips}

Fentanyl test strips allow an individual to detect the presence of fentanyl in their drug supply before using it and can, therefore, allow the individual to adjust their use based on the information. Studies show a high desire for increased access to these testing strips among people who purchase illicit opioids on the street $[76,77]$. In addition, a study by Peiper et al. demonstrated the willingness of people to use them $(81 \%$ of 125 individuals used their supplied strips) and also showed behavior changes such as decreased fentanyl use, performing tester shots, or snorting rather than injecting correlated with use of the strips [78••]. While the use of fentanyl test strips has been increasing as a primary prevention strategy, there is still limited evidence for its ability to decrease overdose risk and fentanyl use. Additionally, while they may be useful for those interested in avoiding inadvertent fentanyl use, detection strips may not be useful in preventing PWUO who prefer fentanyl from seeking out and using the drug.

\section{Drug Sampling Methods}

Because many PWUO do not have access to fentanyl test strips, many have adopted a variety of more informal methods to discern the presence of fentanyl in their drugs. For example, a qualitative study of 91 people who inject heroin and eight people who snort heroin, distributed among five geographically distant states, identified multiple methods of heroin examining and sampling [79]. Examination methods before taking the drug included visual appearance and examination of color, smell and taste of the drug. Sampling methods included noninjection methods (snorting, tasting, smoking), partial injections, and receiving feedback from others after observing someone with a higher tolerance use the drug first. Partial injections allowed the participants to test the drug before committing to the full dose and included slow shots (injecting a portion of the drug and keeping the needle at the site of injection to either continue the injection or later withdraw) and tester shots (dividing the dose up into separate injections). A multisite, cross sectional study performed by Rouhani et al. found that $84 \%$ of participants $(N=334)$ expressed concern about fentanyl in their drug supply [80]. In this same sample, almost $60 \%$ ( $n=196)$ suspected fentanyl in the drugs they planned on using, and $39 \%$ reported participating in harm reduction behavior if they suspected their drugs were contaminated with fentanyl. Specifically, some participants who suspected fentanyl contamination used less drug or did not use the drug at all, and others used a tester shot or used the drug more slowly. While there was a wide variety of harm reduction behaviors employed, this study demonstrated that there are individuals willing to alter their patterns of drug use based on the perceived presence of fentanyl. However, it is important to 
note that the prevalence of these behaviors in a wider population is unknown, as well as the true effectiveness of these behaviors in harm reduction. It is also important to note that this study was performed before fentanyl testing strips were available.

\section{Awareness and Knowledge of Overdose Risk}

Due to the increased susceptibility to opioid overdose among those who are not fully aware or who are misinformed regarding their personal overdose risk [31, 81-83], identifying and intervening with individuals to help them more accurately assess their personal risk is important. Unfortunately, studies suggest a gap in opioid users' knowledge regarding overdose [34]. Even people who use opioids and have experienced an overdose remain uninformed about overdose prevention and naloxone use, demonstrating key missed opportunities for intervention [31]. One study of 122 opioid overdose survivors, found that participants' perceived risk of overdose was low, with $30 \%$ reporting no likelihood of overdose, and only $13 \%$ reporting a high likelihood of overdose [31]. Further, prescription opioid users tended to see themselves as lower risk than heroin users despite also using opioids with the same and possibly stronger potency [31]. A qualitative study assessing young adults who reported witnessing an unintentional opioid overdose, in the context of mixing drugs, found a common theme in interviews where the study participants attributed the overdose to factors such as the individuals "not being able to handle it" with little reference to the risk of overdose due to mixing drugs [84].

\section{Opportunities for Intervention}

\section{Medication for Opioid Use Disorder}

Medication for opioid use disorder (MOUD; i.e., buprenorphine, methadone, and naltrexone) has several decades of research demonstrating its efficacy for OUD, including its ability to decrease overdose and overall mortality [85]. Studies continue to show MOUD's ability to decrease opioid use [86], and even when obtained illicitly and without a prescription, buprenorphine has been shown to be associated with lower rates of overdose in the opioid using population [87]. Some providers initiate buprenorphine treatment in an outpatient setting, often allowing patients to take their first dose at home, or "unobserved", with minimal adverse events [88]. Additionally, an initial dose of buprenorphine can often be started safely in an emergency or inpatient setting with close follow up for long term care [87]. The American College of Medical Toxicology issued a statement in 2019 supporting the administration of buprenorphine to treat opioid withdrawal, reduce risk of overdose post discharge, and to serve as a "bridge" to begin long term addiction treatment [89]. However, there are barriers to obtaining MOUD. Even if clinicians become certified to prescribe buprenorphine, which involves taking a separate course for certification, they are only able to prescribe them to 30 patients in their first year of eligibility which then gets increased to 100 patients [90]. There are additional limits to medication access with providers often limited in the dose they can prescribe, the form that the medication can be provided in, patient eligibility requirements (i.e., minimum 1-year duration of opioid addiction) and requirements for full in-person workups before prescriptions are written [91-93]. These laws could severely limit access to MOUD, with recent studies demonstrating that federal laws are a main barrier to MOUD treatment for those at risk of overdose $[94,95]$. Recently there have been impactful changes to how providers can become trained to provide MOUD, including the American Society of Addiction Medicine offering training either partially or completely online, especially beneficial during the ongoing COVID-19 crisis [96]. Implementation of feasible training models such as online Extensions for Community Healthcare Outcomes (ECHO) have also been implemented with ongoing research analyzing their success and feasibility [97]. Finally, there have been strategies to overcome known barriers to providers attending trainings. For instance, time restraints related to clinical duties and training are an often-cited reason for inability to attend, which has been addressed by offering sessions during lunch hours and giving providers continuing medical education credit [97].

Methadone is another commonly used MOUD with high levels of success. A cohort study consisting of 6983 participants demonstrated a decrease in mortality associated with methadone treatment, with a significant increase in all-cause mortality in participants who stopped treatment [98]. Another cohort study of 32,322 participants demonstrated a decrease in both overall and drug-related mortality with methadone maintenance therapy [99]. Naltrexone is a mu-opioid receptor antagonist shown to have comparable reductions in mortality to both methadone and buprenorphine in a retrospective cohort analysis [100], with another retrospective study demonstrating improved outcomes in neonates of pregnant woman treated with naltrexone or buprenorphine relative to methadone [101]. It is important to note that while valuable, these studies have all been observational.

Medically managed opioid withdrawal (i.e., "detox") has high rates of relapse (90\% within a year) with most $(63 \%)$ of 164 inpatients interviewed expressing a desire for medication treatment after discharge [102]. A cohort study with over 30,000 detox patients demonstrated reduced mortality among those who received MOUD after detox as compared to those who did not receive MOUD postdetox, suggesting that linkage to MOUD after detox could be an effective avenue for decreasing overdose after detox discharge [103•]. 
Another avenue for treatment of OUD is the use of psychedelic drugs as primary or adjuvant treatment. A small $(n=78)$ RCT performed in 1973 demonstrated increased abstinence rates at 12 months in those who received a high-dose LSD treatment [104]. A meta-analysis performed by Dos Santos et. Al suggested ayahuasca, psilocybin and lysergic acid diethylamide (LSD) may have antiaddictive properties, demonstrated by reduction in smoking/alcohol use, withdrawal symptoms, mood, and consequences of drug use [105]. However none of the studies analyzed were specific to OUD, all had extremely small sample sizes $(N=6-15)$, and half did not include a placebo or control group since they were open label or proof of concept. While there are proposed mechanisms for classically psychedelic drugs to decrease dependence and successful case reports have been published [106], further high-quality research is needed before determining any role these drugs could play in a multifaceted approach to the treatment of OUD [107].

\section{Peer Recovery Coaching Postopioid Overdose}

Peer recovery coaching provides bedside support in hospital emergency departments (EDs) and other settings for individuals who experienced an opioid overdose, and peer recovery coaching is becoming common across the United States [108]. The purpose of peer recovery coaching postopioid overdose is to provide social support to isolated individuals, to help link individuals who have overdosed to treatment for substance use and mental health disorders, and to prevent repeat overdoses. Pilot studies of peer recovery coaching interventions in hospital emergency departments targeting people who have experienced an opioid overdose have been performed. One study ( $n=75$ ) demonstrated a much greater likelihood of linkage to programs that provide MOUD (20x more likely) and longer retention in MOUD treatment among people who overdosed and received peer recovery coaching as compared to those who overdosed and received a passive referral to community substance abuse treatment programs [109]. A study of 1329 emergency department peer coaching contacts in Rhode Island demonstrated high rates of patients receiving naloxone training by peer recovery coaches $(88.7 \%)$ and agreeing to continue contact with a peer recovery coach $(86.8 \%)$ [110 ]. Welch et al. demonstrated a $74 \%$ enrollment rate $(N=876)$ in a program linking peer recovery support to overdose survivors for 90 days [111]. A retrospective study analyzing 555 patients admitted to an ED for overdose or who the ED provider documented as having opioid misuse/opioid use disorder reported high rates of peer recovery coach consultation $(85.5 \%)$ and distribution of take-home naloxone (95.2\%) [112]. While these pilot studies demonstrate the feasibility of these programs, to date, no randomized control trial has been completed demonstrating the results of these interventions on patient outcomes. However, there are several protocols for such ongoing trials that have been published $[113,114]$. The evaluation of peer recovery interventions is necessary to optimize their effectiveness.

\section{Technology and Overdose Prevention}

In the current age of technology, it is inevitable that methods for harm reduction, utilizing technology, will appear. Innovative wearable devices that can detect physiologic changes signaling overdose are being developed that could theoretically assist in harm reduction, allowing practitioners to intervene earlier than currently possible [115]. Similarly, devices have been tested to monitor for opioid withdrawal [116] and proof-of-concept smartphones developed that can detect opioid overdose [117]. There have also been design proposals for devices that can monitor for overdose and deliver naloxone to the wearer [118]. This is an exciting new field with promise for innovation in the field of harm reduction.

\section{Conclusion}

The current opioid epidemic is a quickly evolving crisis, with both old and new factors influencing opioid overdose events and outcomes. The variables responsible for increased fentanyl in the drug market are multifactorial. While market forces may account for much of the proliferation of fentanyl in the last several years, there is a small but growing body of evidence which suggests that some PWUO prefer and actively seek out fentanyl. Continued research identifying the drivers of fentanyl use will allow for proper targeting of fentanyl education and interventions. Similarly, while naloxone distribution to the general public continues to increase, little is known about the responses of PWUO to the increased availability of naloxone. The few studies exploring possible naloxone-related risk-taking, or perceptions and behaviors surrounding naloxone-induced withdrawal, for example, have been qualitative studies with limited generalizability. More large-scale studies exploring these issues are clearly needed in order to enhance naloxone's safety without limiting its accessibility to the public. Finally, more research is needed on the variety of harm reduction techniques proposed to reduce opioid overdose. Supervised injection facilities are not yet available in the United States, for example, but data from other countries suggest that these safe using spaces can be an effective harm reduction tool, and studies from within the United States suggest that PWUO would be willing to take advantage of them. Further, studies suggest that PWUO tend to underestimate their overdose risk or not be aware of their overdose risk factors, putting them in a situation where they may be participating in behaviors that increase their risk without realizing it. This indicates a need for research providing targeted interventions for this high-risk group. Pilot studies of peer 
recovery coaching to link PWUO to treatment postopioid overdose have been promising, and RCTs are currently underway to test their effectiveness. Overall, the continuation of innovative research into identifying and monitoring overdose risk factors and analyzing the effectiveness of interventions will remain pivotal in combatting overdose morbidity and mortality.

\section{References}

Papers of particular interest, published recently, have been highlighted as:

- Of importance

•- Of major importance

1. Rudd RA, Paulozzi LJ, Bauer MJ, Burleson RW, Carlson RE, Dao D, et al. Increases in heroin overdose deaths - 28 states, 2010 to 2012. MMWR. Morb Mortal Wkly Rep. 2014;63(39): 849.

2. CDC/NCHS, National Vital Statistics System, Mortality. CDC WONDER. Atlanta: US Department of Health and Human Services, CDC; 2018. https://wonder.cdc.gov

3. Park TW, Lin LA, Hosanagar A, Kogowski A, Paige K, Bohnert AS. Understanding risk factors for opioid overdose in clinical populations to inform treatment and policy. J Addict Med. 2016;10(6):369-81.

4. Yarborough BJ, Stumbo SP, Janoff SL, Yarborough MT, McCarty D, Chilcoat HD, et al. Understanding opioid overdose characteristics involving prescription and illicit opioids: a mixed methods analysis. Drug Alcohol Depend. 2016;167:49-56.

5. Wilson N. Drug and opioid-involved overdose deaths - United States, 2017-2018. MMWR Morb Mortal Wkly Rep. 2020;69.

6. Vivolo-Kantor AM, Seth P, Gladden RM, Mattson CL, Baldwin GT, Kite-Powell A, et al. Vital signs: trends in emergency department visits for suspected opioid overdoses-United States, July 2016-September 2017. Morb Mortal Wkly Rep. 2018;67(9):279-85.

7. Chen Q, Larochelle MR, Weaver DT, Lietz AP, Mueller PP, Mercaldo S, et al. Prevention of prescription opioid misuse and projected overdose deaths in the United States. JAMA Netw Open. 2019;2(2):e187621.

8. Felbab-Brown V, Caulkins JP, Graham C, Humphreys K, Pacula RL, Pardo B, Reuter P, Stein BD, Wise PH. The opioid crisis in America.

9. Kilmer G. EMS calls show $54 \%$ spike in drug overdoses, $80 \%$ increase in suicide attempts. Urban Milwaukee. 2020. Accessed online 12/6/2020. https://urbanmilwaukee.com/2020/05/07/emscalls-show-54-spike-in-drug-overdoses-80-increase-in-suicideattempts/.

10. Kovac M. OD deaths surge on a weekend again. The Columbus Dispatch. 2020 May 11. Accessed online 12/6/2020. https://www. dispatch.com/news/20200511/od-deaths-surge-on-weekendagain.

11. Armenian P, Vo KT, Barr-Walker J, Lynch KL. Fentanyl, fentanyl analogs and novel synthetic opioids: a comprehensive review. Neuropharmacology. 2018;134:121-32.

12. Drug Enforcement Administration. DEA Intelligence Brief. Counterfeit prescription pills containing fentanyls: a global threat. DEA-DCT-DIB-021-16. July 2016. Available at: https://www.
dea.gov/docs/Counterfeit\%20Prescription\%20Pills.pdf. Accessed 5 Aug 2017.

13. Alpert A, Powell D, Pacula RL. Supply-side drug policy in the presence of substitutes: Evidence from the introduction of abusedeterrent opioids. Am Econ J Econ Pol. 2018;10(4):1-35.

14. Patrick SW, Fry CE, Jones TF, Buntin MB. Implementation of prescription drug monitoring programs associated with reductions in opioid-related death rates. Health Aff. 2016;35(7):1324-32.

15. Anderson DT, Muto JJ. Duragesic $\AA$ transdermal patch: postmortem tissue distribution of fentanyl in 25 cases. J Anal Toxicol. 2000;24(7):627-34.

16. Helander A, Bäckberg M, Signell P, Beck O. Intoxications involving acrylfentanyl and other novel designer fentanyls - results from the Swedish STRIDA project. Clin Toxicol. 2017;55(6):589-99.

17. Sutter ME, Gerona RR, Davis MT, Roche BM, Colby DK, Chenoweth JA, et al. Fatal fentanyl: one pill can kill. Acad Emerg Med. 2017;24(1):106-13.

18. Somerville NJ, O'Donnell J, Gladden RM, et al. Characteristics of fentanyl overdose-Massachusetts, 2014-2016. MMWR Morb Mortal Wkly Rep. 2017;66(14):382-6.

19. Drug Enforcement Administration. National heroin threat assessment summary-updated. Washington DC: Department of Justice; 2016.

20. New Jersey State Police. Office of drug monitoring and analysis. 2020. Fentanyl trends in New Jersey, January 1, 2015-December 31, 2019.

21. Bohnert AS, Ilgen MA. Understanding links among opioid use, overdose, and suicide. N Engl J Med. 2019;380(1):71-9.

22. Stein MD, Kenney SR, Anderson BJ, Bailey GL. Perceptions about fentanyl-adulterated heroin and overdose risk reduction behaviors among persons seeking treatment for heroin use. J Subst Abus Treat. 2019;104:144-7. This is an important study demonstrating the prevalence of perceived fentanyl contamination and suggests that increased perceived fentanyl in supply did not coincide with increased overdose reduction behaviors.

23. Hempstead K, Yildirim EO. Supply-side response to declining heroin purity: fentanyl overdose episode in New Jersey. Health Econ. 2014;23(6):688-705.

24. Cicero TJ, Ellis MS, Surratt HL, Kurtz SP. The changing face of heroin use in the United States: a retrospective analysis of the past 50 years. JAMA Psychiatry. 2014;71(7):821-6.

25. Beletsky L, Davis CS. Today's fentanyl crisis: Prohibition's Iron Law, revisited. Int J Drug Policy. 2017 Aug 1;46:156-9.

26.• Morales KB, Park JN, Glick JL, Rouhani S, Green TC, Sherman SG. Preference for drugs containing fentanyl from a crosssectional survey of people who use illicit opioids in three United States cities. Drug Alcohol Depend. 2019;204:107547. This was one of the earliest and largest studies demonstrating a significant portion of the population that preferred fentanyl.

27. Daniulaityte R, Carlson RR, Juhascik MP, Strayer KE, Sizemore IE. Street fentanyl use: experiences, preferences, and concordance between self-reports and urine toxicology. Int J Drug Policy. 2019;71:3-9.

28. Kertesz SG. Turning the tide or riptide? The changing opioid epidemic. Subst Abus. 2017;38(1):3-8.

29. Park JN, Weir BW, Allen ST, Chaulk P, Sherman SG. Fentanylcontaminated drugs and non-fatal overdose among people who inject drugs in Baltimore, MD. Harm Reduct J. 2018;15(1):1-8.

30. Garg RK, Fulton-Kehoe D, Franklin GM. Patterns of opioid use and risk of opioid overdose death among Medicaid patients. Med Care. 2017;55(7):661-8.

31. Frank D, Mateu-Gelabert P, Guarino H, Bennett A, Wendel T, Jessell L, et al. High risk and little knowledge: overdose experiences and knowledge among young adult nonmedical prescription opioid users. Int J Drug Policy. 2015;26(1):84-91. 
32. Sehgal N, Manchikanti L, Smith HS. Prescription opioid abuse in chronic pain: a review of opioid abuse predictors and strategies to curb opioid abuse. Pain Phys. 2012;15(3 Suppl):ES67-92.

33. Oquendo MA, Volkow ND. Suicide: a silent contributor to opioidoverdose deaths. N Engl J Med. 2018;378(17):1567-9.

34. Connery HS, Taghian N, Kim J, Griffin M, Rockett IR, Weiss RD, et al. Suicidal motivations reported by opioid overdose survivors: a cross-sectional study of adults with opioid use disorder. Drug Alcohol Depend. 2019;205:107612. This study identified a high rate of misconceptions about overdose risk in PWUO even after overdose, suggesting a need for targeted interventions in this population, as well as a description of the need to further examine suicidal thoughts and behaviors in PWUO.

35. Prescription Drug Abuse Policy System. Naloxone overdose prevention laws. Philadelphia: Prescription Drug Abuse Policy System; 2017. http://pdaps.org/datasets/laws-regulatingadministration-of-naloxone-1501695139. Accessed online 12/6/ 2020

36. Davis C, Carr D. State legal innovations to encourage naloxone dispensing. J Am Pharm Assoc. 2017;57(2):S180-4.

37. Wheeler E, Jones TS, Gilbert MK, Davidson PJ. Opioid overdose prevention programs providing naloxone to laypersons - United States, 2014. MMWR Morb Mortal Wkly Rep. 2015;64(23):631.

38. Jones JD, Campbell A, Metz VE, Comer SD. No evidence of compensatory drug use risk behavior among heroin users after receiving take-home naloxone. Addict Behav. 2017;71:104-6. This study demonstrated decreased heroin use and no change in severity of drug use after naloxone training, helping to start building an argument against widespread increases in risky drug use after naloxone training.

39. Nielsen S, Peacock A, Lintzeris N, Bruno R, Larance B, Degenhardt L. Knowledge of opioid overdose and attitudes to supply of take-home naloxone among people with chronic noncancer pain prescribed opioids. Pain Med. 2018;19(3):53340.

40. Mueller SR, Koester S, Glanz JM, Gardner EM, Binswanger IA. Attitudes toward naloxone prescribing in clinical settings: a qualitative study of patients prescribed high dose opioids for chronic non-cancer pain. J Gen Intern Med. 2017;32(3):277-83.

41. Abouk R, Pacula RL, Powell D. Association between state laws facilitating pharmacy distribution of naloxone and risk of fatal overdose. JAMA Intern Med. 2019;179(6):805-11.

42.• McClellan C, Lambdin BH, Ali MM, Mutter R, Davis CS, Wheeler E, et al. Opioid-overdose laws association with opioid use and overdose mortality. Addict Behav. 2018;86:90-5. This study looked at a relatively long period of national survey data that suggests increased naloxone access led to decreased opioid overdose deaths with no increase in opioid use.

43. Langham S, Wright A, Kenworthy J, Grieve R, Dunlop WC. Costeffectiveness of take-home naloxone for the prevention of overdose fatalities among heroin users in the United Kingdom. Value Health. 2018;21(4):407-15.

44. Ruhm CJ. Drug poisoning deaths in the United States, 19992012: a statistical adjustment analysis. Popul Health Metrics. 2016;14(1):2

45. Katzman JG, Takeda MY, Bhatt SR, Balasch MM, Greenberg N, Yonas H. An innovative model for naloxone use within an OTP setting: a prospective cohort study. J Addict Med. 2018;12(2): 113-8.

46. Siegler A, Huxley-Reicher Z, Maldjian L, Jordan R, Oliver C, Jakubowski A, et al. Naloxone use among overdose prevention trainees in New York City: A longitudinal cohort study. Drug Alcohol Depend. 2017;179:124-30.

47. Katzman JG, Takeda MY, Greenberg N, Balasch MM, Alchbli A, Katzman WG, et al. Association of take-home naloxone and opioid overdose reversals performed by patients in an opioid treatment program. JAMA Netw Open. 2020;3(2):e200117.

48. Naumann RB, Durrance CP, Ranapurwala SI, Austin AE, Proescholdbell S, Childs R, et al. Impact of a community-based naloxone distribution program on opioid overdose death rates. Drug Alcohol Depend. 2019;204:107536.

49. Walley AY, Xuan Z, Hackman HH, Quinn E, Doe-Simkins M, Sorensen-Alawad A, et al. Opioid overdose rates and implementation of overdose education and nasal naloxone distribution in Massachusetts: interrupted time series analysis. Bmj. 2013;31: 346.

50. Hilton MT. Mixed feelings about naloxone: it saves lives, but at what cost. Medscape Emerg Med. 2018.

51. On the front lines of opioid epidemic, U.S. police split on Narcan. CBS News. December 19, 2017. Accessed online 12/6/2020 https://www.cbsnews.com/news/narcan-police-split-opioidepidemic/.

52. Heavey SC, Chang YP, Vest BM, Collins RL, Wieczorek W, Homish GG. 'I have it just in case'-naloxone access and changes in opioid use behaviours. Int J Drug Policy. 2018;51:27-35.

53. Kline A, Mattern D, Cooperman N, Dooley-Budsock P, Williams JM, Borys S. "A blessing and a curse:" opioid users' perspectives on naloxone and the epidemic of opioid overdose. Subst Use Misuse. 2020;55(8):1280-7.

54. Marshall JR, Gassner SF, Anderson CL, Cooper RJ, Lotfipour S, Chakravarthy B. Socioeconomic and geographical disparities in prescription and illicit opioid-related overdose deaths in Orange County, California, from 2010-2014. Subst Abus. 2018.

55. Marco CA, Trautman W, Cook A, Mann D, Rasp J, Perkins O, et al. Naloxone use among emergency department patients with opioid overdose. J Emerg Med. 2018;55(1):64-70.

56. Wagner KD, Valente TW, Casanova M, Partovi SM, Mendenhall BM, Hundley JH, et al. Evaluation of an overdose prevention and response training programme for injection drug users in the Skid Row area of Los Angeles, CA. Int J Drug Policy. 2010;21(3):186-93.

57. Rzasa Lynn R, Galinkin JL. Naloxone dosage for opioid reversal: current evidence and clinical implications. Ther Adv Drug Saf. 2018;9(1):63-88.

58. Vanky E, Hellmundt L, Bondesson U, Eksborg S, Lundeberg S. Pharmacokinetics after a single dose of naloxone administered as a nasal spray in healthy volunteers. Acta Anaesthesiol Scand. 2017;61(6):636-40.

59. Bell A, Bennett AS, Jones TS, Doe-Simkins M, Williams LD. Amount of naloxone used to reverse opioid overdoses outside of medical practice in a city with increasing illicitly manufactured fentanyl in illicit drug supply. Subst Abus. 2018.

60. Neale J, Strang J. Naloxone-does over-antagonism matter? Evidence of iatrogenic harm after emergency treatment of heroin/opioid overdose. Addiction. 2015;110(10):1644-52.

61. Kerensky T, Walley AY. Opioid overdose prevention and naloxone rescue kits: what we know and what we don't know. Addict Sci Clin Pract. 2017;12(1):4.

62. Martino JG, Smith SR, Rafie S, Rafie S, Marienfeld C. Physician and pharmacist: attitudes, facilitators, and barriers to prescribing naloxone for home rescue. Am J Addict. 2020;29(1):65-72.

63. Gupta R, Shah ND, Ross JS. The rising price of naloxone-risks to efforts to stem overdose deaths. N Engl J Med. 2016;375(23): 2213-5.

64. Friedman SR, West BS, Pouget ER, Hall HI, Cantrell J, Tempalski B, et al. Metropolitan social environments and pre-HAART/ HAART era changes in mortality rates (per 10,000 adult residents) among injection drug users living with AIDS. PLoS One. 2013;8(2):e57201.

65. Heavey SC, Burstein G, Moore C, Homish GG. Overdose education and naloxone distribution program attendees: who attends, 
what do they know, and how do they feel? J Publ Health Manag Pract. 2018;24(1):63-8.

66. Centers for Disease Control and Prevention. Opioid overdose: understanding the epidemic Available at: https://www.cdc.gov/ drugoverdose/epidemic/index.html. Accessed 8 Febr 2019.

67. BC Coroners Service. Illicit drug overdose deaths in BC: January 1, 2008-December 31, 2018. British Columbia. 2019. Accessed online 12/6/2020. https://www2.gov.bc.ca/assets/gov/birthadoption-death-marriage-and-divorce/deaths/coroners-service/ statistical/illicit-drug.pdf.

68. Vaughan AD. Improving estimates of the quantity and quality of persons involved in the opioid crisis. In: Geographies of behavioural health, crime, and disorder. Cham: Springer; 2020. p. $37-$ 57.

69. Bardwell G, Boyd J, Kerr T, McNeil R. Negotiating space \& drug use in emergency shelters with peer witness injection programs within the context of an overdose crisis: a qualitative study. Health Place. 2018;53:86-93.

70. Potier C, Laprévote V, Dubois-Arber F, Cottencin O, Rolland B. Supervised injection services: what has been demonstrated? A systematic literature review. Drug Alcohol Depend. 2014;145: 48-68.

71. Hedrich D. European report on drug consumption rooms. Luxembourg: Office for Official Publications of the European Communities; 2004.

72. USC Department of Nursing. Supervised injection sites are coming to the United States. Here's what you should know. May 2, 2019. Blog. Accessed 12/26/2020 https://nursing.usc.edu/blog/ supervised-injection-sites/.

73. Vipler S, Hayashi K, Milloy MJ, Wood E, Nosova E, Kerr T, et al. Use of withdrawal management services among people who use illicit drugs in Vancouver, Canada. Subst Abus Treat Prev Policy. 2018;13(1):27.

74. Park JN, Sherman SG, Rouhani S, Morales KB, McKenzie M, Allen ST, et al. Willingness to use safe consumption spaces among opioid users at high risk of fentanyl overdose in Baltimore, Providence, and Boston. J Urban Health. 2019;96(3): 353-66.

75. León C, Cardoso L, Mackin S, Bock B, Gaeta JM. The willingness of people who inject drugs in Boston to use a supervised injection facility. Subst Abus. 2018;39(1):95-101.

76. Krieger MS, Goedel WC, Buxton JA, Lysyshyn M, Bernstein E, Sherman SG, et al. Use of rapid fentanyl test strips among young adults who use drugs. Int J Drug Policy. 2018;61:52-8.

77. Krieger MS, Yedinak JL, Buxton JA, Lysyshyn M, Bernstein E, Rich JD, et al. High willingness to use rapid fentanyl test strips among young adults who use drugs. Harm Reduct J. 2018;15(1):7.

78.• Peiper NC, Clarke SD, Vincent LB, Ciccarone D, Kral AH, Zibbell JE. Fentanyl test strips as an opioid overdose prevention strategy: findings from a syringe services program in the Southeastern United States. Int J Drug Policy. 2019;63:122-8. One of the first relatively large studies examining fentanyl testing strips as a harm reduction technique, demonstrating their potential effectiveness.

79. Mars SG, Ondocsin J, Ciccarone D. Toots, tastes and tester shots: user accounts of drug sampling methods for gauging heroin potency. Harm Reduct J. 2018;15(1):26.

80. Rouhani S, Park JN, Morales KB, Green TC, Sherman SG. Harm reduction measures employed by people using opioids with suspected fentanyl exposure in Boston, Baltimore, and Providence. Harm Reduct J. 2019;16(1):39.

81. Mcgregor C, Darke S, Ali R, Christie P. Experience of non-fatal overdose among heroin users in Adelaide, Australia: circumstances and risk perceptions. Addiction. 1998;93(5):701-11.

82. Neira-León M, Barrio G, Brugal MT, de la Fuente L, Ballesta R, Bravo MJ, et al. Project Itinere Group. Do young heroin users in
Madrid, Barcelona and Seville have sufficient knowledge of the risk factors for unintentional opioid overdose? J Urban Health. 2006;83(3):477-96.

83. Rowe C, Santos GM, Behar E, Coffin PO. Correlates of overdose risk perception among illicit opioid users. Drug Alcohol Depend. 2016;159:234-9.

84. Yedinak JL, Kinnard EN, Hadland SE, Green TC, Clark MA, Marshall BD. Social context and perspectives of non-medical prescription opioid use among young adults in Rhode Island: a qualitative study. Am J Addict. 2016;25(8):659-65.

85. Sordo L, Barrio G, Bravo MJ, Indave BI, Degenhardt L, Wiessing $\mathrm{L}$, et al. Mortality risk during and after opioid substitution treatment: systematic review and meta-analysis of cohort studies. BMJ. 2017;26:357.

86. Nielsen S, Larance B, Lintzeris N. Opioid agonist treatment for patients with dependence on prescription opioids. Jama. 2017;317(9):967-8.

87. Carlson RG, Daniulaityte R, Silverstein SM, Nahhas RW, Martins SS. Unintentional drug overdose: is more frequent use of nonprescribed buprenorphine associated with lower risk of overdose? Int J Drug Policy. 2020;79:102722.

88. Lee JD, Vocci F, Fiellin DA. Unobserved "home" induction onto buprenorphine. J Addict Med. 2014;8(5):299-308.

89. Wax PM, Stolbach AI, Schwarz ES, Warrick BJ, Wiegand TJ, Nelson LS. ACMT position statement: buprenorphine administration in the emergency department. J Med Toxicol. 2019;15(3): $215-6$.

90. United States Code, 2019b United States Code 21 U.S.C. § 823(g)(2)(B) (2019).

91. United States Code, 2019e United States Code 42 C.F.R. § 8.12(e) (2019).

92. United States Code, $2019 f$ United States Code 42 C.F.R. $\S$ 8.12(h)(3) (2019).

93. United States Code, 2019g United States Code 42 C.F.R. § 8.12(i) (2019).

94. Olfson M, Zhang VS, Schoenbaum M, King M. Trends in buprenorphine treatment in the United States, 2009-2018. Jama. 2020;323(3):276-7.

95. Davis CS, Carr DH. Legal and policy changes urgently needed to increase access to opioid agonist therapy in the United States. Int J Drug Policy. 2019;73:42-8.

96. Waiver Qualifying Training. American Society of Addiction Medicine. [accessed 2021Feb18]. Available from: https://www. asam.org/education/live-online-cme/waiver-qualifying-training.

97. Salvador JG, Bhatt SR, Jacobsohn VC, Maley LA, Alkhafaji RS, Rishel Brakey H, et al. Feasibility and acceptability of an online $\mathrm{ECHO}$ intervention to expand access to medications for treatment of opioid use disorder, psychosocial treatments and supports. Subst Abus. 2020;1-8.

98. Cousins G, Boland F, Courtney B, Barry J, Lyons S, Fahey T. Risk of mortality on and off methadone substitution treatment in primary care: a national cohort study. Addiction. 2016;111(1):7382.

99. Evans E, Li L, Min J, Huang D, Urada D, Liu L, et al. Mortality among individuals accessing pharmacological treatment for opioid dependence in California, 2006-10. Addiction. 2015;110(6): 996-1005.

100. Kelty E, Joyce D, Hulse G. A retrospective cohort study of mortality rates in patients with an opioid use disorder treated with implant naltrexone, oral methadone or sublingual buprenorphine. Am J Drug Alcohol Abus. 2019;45(3):285-91.

101. Kelty E, Hulse G. A retrospective cohort study of birth outcomes in neonates exposed to naltrexone in utero: a comparison with methadone-, buprenorphine-and non-opioid-exposed neonates. Drugs. 2017;77(11):1211-9. 
102. Bailey GL, Herman DS, Stein MD. Perceived relapse risk and desire for medication assisted treatment among persons seeking inpatient opiate detoxification. J Subst Abus Treat. 2013;45(3): 302-5.

103. Walley AY, Lodi S, Li Y, Bernson D, Babakhanlou-Chase H, Land T, et al. Association between mortality rates and medication and residential treatment after in-patient medically managed opioid withdrawal: a cohort analysis. Addiction. 2020. This is a cohort study with a large sample demonstrating a significant benefit to MOUD after detox vs. detox alone, which could lead to more favorable outcomes for individuals seeking treatment.

104. Savage C, McCabe OL. Residential psychedelic (LSD) therapy for the narcotic addict: a controlled study. Arch Gen Psychiatry. 1973;28(6):808-14.

105. Dos Santos RG, Osório FL, Crippa JA, Riba J, Zuardi AW, Hallak JE. Antidepressive, anxiolytic, and antiaddictive effects of ayahuasca, psilocybin and lysergic acid diethylamide (LSD): a systematic review of clinical trials published in the last 25 years. Ther Adv Psychopharmacol. 2016;6(3):193-213.

106. Cloutier-Gill L, Wood E, Millar T, Ferris C, Eugenia SM. Remission of severe opioid use disorder with ibogaine: a case report. J Psychoactive Drugs. 2016;48(3):214-7.

107. Argento E, Tupper KW, Socias ME. The tripping point: the potential role of psychedelic-assisted therapy in the response to the opioid crisis. Int J Drug Policy. 2019;66:80-1.

108. McGuire AB, Powell KG, Treitler PC, Wagner KD, Smith KP, Cooperman N, et al. Emergency department-based peer support for opioid use disorder: emergent functions and forms. J Subst Abus Treat. 2020;108:82-7.

109. Scott CK, Dennis ML, Grella CE, Nicholson L, Sumpter J, Kurz $\mathrm{R}$, et al. Findings from the recovery initiation and management after overdose (RIMO) pilot study experiment. J Subst Abus Treat. 2020;108:65-74.

110. Waye KM, Goyer J, Dettor D, Mahoney L, Samuels EA, Yedinak JL, et al. Implementing peer recovery services for overdose prevention in Rhode Island: an examination of two outreach-based approaches. Addict Behav. 2019;89:85-91. This study showed that overdose survivors are willing to use peer delivered interventions in the ED, keep in contact with their peer recovery coach, and receive naloxone training.

111. Welch AE, Jeffers A, Allen B, Paone D, Kunins HV. Relay: a peer-delivered emergency department-based response to nonfatal opioid overdose. Am J Public Health. 2019;109(10):1392-5.

112. Samuels EA, Baird J, Yang ES, Mello MJ. Adoption and utilization of an emergency department naloxone distribution and peer recovery coach consultation program. Acad Emerg Med. 2019;26(2):160-73.

113. Orkin A, Campbell D, Handford C, Hopkins S, Klaiman M, Leece $\mathrm{P}$, et al. Protocol for a mixed-methods feasibility study for the surviving opioid overdose with naloxone education and resuscitation (SOONER) randomized control trial. BMJ Open. 2019;9(11).

114. Goedel WC, Marshall BD, Samuels EA, Brinkman MG, Dettor D, Langdon $\mathrm{KJ}$, et al. Randomised clinical trial of an emergency department-based peer recovery support intervention to increase treatment uptake and reduce recurrent overdose among individuals at high risk for opioid overdose: study protocol for the navigator trial. BMJ Open. 2019;9(11).

115. Carreiro S, Wittbold K, Indic P, Fang H, Zhang J, Boyer EW. Wearable biosensors to detect physiologic change during opioid use. J Med Toxicol. 2016;12(3):255-62.

116. Kulman E, Chapman B, Venkatasubramanian K, Carreiro S. Identifying opioid withdrawal using wearable biosensors. In Proceedings of the Annual Hawaii International Conference on System Sciences. Annual Hawaii International Conference on System Sciences (Vol. 54, p. 3583). NIH Public Access; 2021.

117. Nandakumar R, Gollakota S, Sunshine JE. Opioid overdose detection using smartphones. Sci Transl Med. 2019 Jan;9:11(474).

118. Gilbert M. OneUp: a lifesaving wearable device. In Computer human interaction conference, Toronto, Canada; 2014. Paper retrieved from http://courses.media.mit.edu/2014spring/mass64/ UPLOADS/FinalPaper-Gilbert.pdf.

Publisher's Note Springer Nature remains neutral with regard to jurisdictional claims in published maps and institutional affiliations. 